Dispersion in nulling interferometry for exoplanet detection: experimental validation

This article has been downloaded from IOPscience. Please scroll down to see the full text article.

2009 J. Opt. A: Pure Appl. Opt. 11015510

(http://iopscience.iop.org/1464-4258/11/1/015510)

View the table of contents for this issue, or go to the journal homepage for more

Download details:

IP Address: 131.180.130.109

The article was downloaded on 08/08/2011 at 10:40

Please note that terms and conditions apply. 


\title{
Dispersion in nulling interferometry for exoplanet detection: experimental validation
}

\author{
J F P Spronck, J W N Los and S F Pereira \\ Optics Research Group, Faculty of Applied Sciences, Delft University of Technology, \\ Lorentzweg 1, 2628 CJ Delft, The Netherlands \\ E-mail: S.F.Pereira@tudelft.nl
}

Received 9 September 2008, accepted for publication 3 December 2008

Published 30 December 2008

Online at stacks.iop.org/JOptA/11/015510

\begin{abstract}
It is well known that dispersion affects the performance of a wide-band nulling interferometer, since it induces wavelength-dependent phase differences between the arms of the interferometer. This property is used to create achromatic phase shift by combining several dielectric plates. In this paper, we experimentally validate the use of a single glass plate in order to optimize the rejection ratio of a nulling interferometer.
\end{abstract}

Keywords: nulling interferometry, exoplanets, interferometry, astronomical optics

(Some figures in this article are in colour only in the electronic version)

\section{Introduction}

Despite all the efforts made in searching for extra-solar planets, or exoplanets, in the last decade, direct detection of an Earth-like exoplanet remains very challenging. The reason for this is the combination of a huge brightness contrast between the star and the planet (10 $10^{6}$ in the best case) and a small angular separation (typically $0.1 \mathrm{arcsec}$ ). One possible candidate technique for meeting this challenge is called nulling interferometry [1]; this will be considered in this paper.

Nulling interferometry is a technique used to cancel the light coming from an on-axis star by means of destructive interference between several beams. Due to the off-axis angular position of a planet, light coming from such a planetary companion would experience an additional phase difference between the beams, which would lead to a (partially) constructive interference for the companion. In order to detect an Earth-like exoplanet, the ratio between the intensities corresponding to constructive and destructive interference, the so-called rejection ratio, should be of the order of $10^{6}$. In this paper, we will discuss nulling interferometry in the specific context of the Darwin/TPF space mission [2, 3], dedicated to direct exoplanet detection via nulling interferometry.

An additional requirement is that this high rejection ratio should be achieved for every wavelength in a wide spectral band (6-18 $\mu \mathrm{m}$ or even wider [4]). Indeed, such a broad band is needed in order to optimally exploit the photon flux coming from the planet and in order to obtain spectral information from the eventual planet atmosphere. To do so, most current nulling interferometers use an achromatic phase shifter [5-8]. Unfortunately, manufacturing and using such phase shifters is technically challenging, since phase variations of typically 1 mrad or less across the spectral band are required to achieve the desired performance. Note that there are also a family of nulling interferometers that make use of chromatic phase shifters such as delay lines $[9,10]$.

Dispersion affects the performance of a wide-band nulling interferometer by inducing wavelength-dependent phase differences between the arms of the interferometer. It is already well known that dispersion can be used to achieve an achromatic phase shift by combining multiple plates with different wavelength-dependent refractive indices [11, 12]. However, single dispersive plates can also lead to a high rejection ratio in a wide spectral band by increasing the number of beams [9]. In this paper, we present an experimental validation of this principle. Note that a similar approach has been used in the Keck interferometer to achieve destructive interference while compensating for dispersion due to unequal path lengths in humid air [13].

This paper is organized as follows. In section 2, we analyse the effect of dispersion on the rejection ratio in the case of $N$-beam nulling interferometers. In section 3 , 


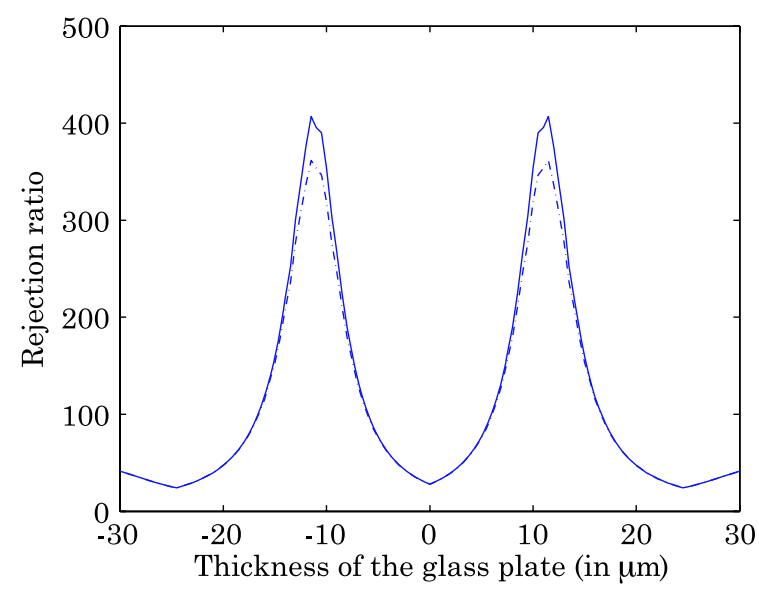

Figure 1. Rejection ratio as a function of the thickness of the introduced glass plate. Solid lines: the spectra of the two beams are identical and equal to the spectrum of the Xe lamp used in our experimental set-up; dash-dotted lines: the spectra of the beams are slightly different, leading to a lower rejection ratio.

we experimentally validate our theoretical predictions with our table-top experimental set-up. Our conclusions are then summarized in section 4 .

\section{Theory and simulations}

In this section, we will briefly show how dispersion can affect the performance of a nulling interferometer. The basic theory has been already presented by Mieremet and Braat [9]. Therefore, in this section, we only briefly discuss these results in the context of the current status of the Darwin mission. The interested reader should refer to [9] for more details. Additionally, we also present an extension of the previously reported results to different numbers of beams and different materials, as well as the tolerances required to achieve the desired rejection ratio.

\subsection{Two-beam interferometer}

We consider two beams of equal amplitudes $a(\lambda)$ with an optical path difference $L$ between them. If the second beam goes through an extra glass plate of thickness $d$ and of refractive index $n(\lambda)$, we can show that the intensity $I(L, d)$ after beam combination is given by

$$
I(L, d)=4 a \int|a(\lambda)|^{2} \cos ^{2}\left\{\frac{\pi}{\lambda}[L+n(\lambda) d]\right\} \mathrm{d} \lambda .
$$

Such intensity as a function of the optical path difference and of the thickness of the glass plate is well known [14]. For a given thickness $d$ of the glass plate, the interference pattern is found by scanning the optical path difference $L$. If we define the rejection ratio $R$ as the ratio between the maximum (constructive interference) and the minimum (destructive interference) of the interference pattern, we have

$$
R(d)=\frac{\max _{L}[I(L, d)]}{\min _{L}[I(L, d)]} .
$$

Table 1. Values of the amplitudes $A_{j}$ and phases $\phi_{j}$ for $N \leqslant 6$.

\begin{tabular}{ccc}
\hline $\mathbf{N}$ & $\mathbf{A}_{\mathbf{1}} \cdots \mathbf{A}_{\mathbf{N}}$ & $\phi_{\mathbf{1}} \cdots \phi_{\mathbf{N}}$ \\
\hline 2 & 11 & $0 \pi$ \\
3 & 121 & $0 \pi 2 \pi$ \\
4 & 1331 & $0 \pi 2 \pi 3 \pi$ \\
5 & 14641 & $0 \pi 2 \pi 3 \pi 4 \pi$ \\
6 & 15101051 & $0 \pi 2 \pi 3 \pi 4 \pi 5 \pi$ \\
\hline
\end{tabular}

In our simulations, we chose the refractive index $n(\lambda)$ of BK7 and for the amplitude $a(\lambda)$, we used the spectrum of the Xe arc visible light source in order to compare with our experimental data. The rejection ratio as a function of the thickness $d$ of the glass plate is depicted in figure 1(a) (solid line). Note that negative values of the thickness $d$ physically mean that the glass plate has been introduced in the first beam instead of the second one. We can see that the rejection ratio is minimal when there is no glass plate $(d=0 \mu \mathrm{m})$ and is maximal when the thickness of the glass plate is around $12 \mu \mathrm{m}$. In order to compare the theory with the experiment, we also plot in figure 1 (dash-dotted line) the rejection ratio when the two beams have slightly different spectra $\left(a_{1}(\lambda) \neq a_{2}(\lambda)\right)$ as is the case in our experimental set-up $[15,16]$. In this case, the rejection ratio has the same overall shape but the peak values are lower.

We can show that the maximal rejection ratio corresponds to the thickness $d$ for which the phase difference between the two beams is equal to $\pi$ quasi-achromatically (to the first order). We see that the rejection ratio is theoretically limited to 400 , which is very low for exoplanet detection. However, as we will see in the next section, increasing the number of telescopes allows high rejection ratios in a wide spectral band.

\section{2. $N$-beam interferometers}

Since the number of telescopes for the Darwin mission changed from six to three and currently to four, it is important to study the influence of the number of beams on the rejection ratio. In this section, we will consider $N$-beam interferometers $(N \leqslant 6)$ and will also analyse the influence of the spectral bandwidth.

Consider the family of interferometers proposed by Mieremet [9] in order to lower the requirements in terms of achromaticity. Given a certain phase shift error introduced by the phase shifter, this family of interferometers leads to the optimal rejection ratio. These interferometers have $N$ beams with amplitudes $A_{j}$ and desired phases $\phi_{j}$ given by

$$
\begin{aligned}
& A_{j}=\left(\begin{array}{c}
N-1 \\
j-1
\end{array}\right), \\
& \phi_{j}=(j-1) \frac{2 \pi}{N} .
\end{aligned}
$$

These amplitudes and phases are summarized in table 1 for $(N \leqslant 6)$.

Each beam goes through a glass plate, whose thickness is optimally chosen to attain as closely as possible the desired phase over the whole spectral band. The spectrum of the beams 

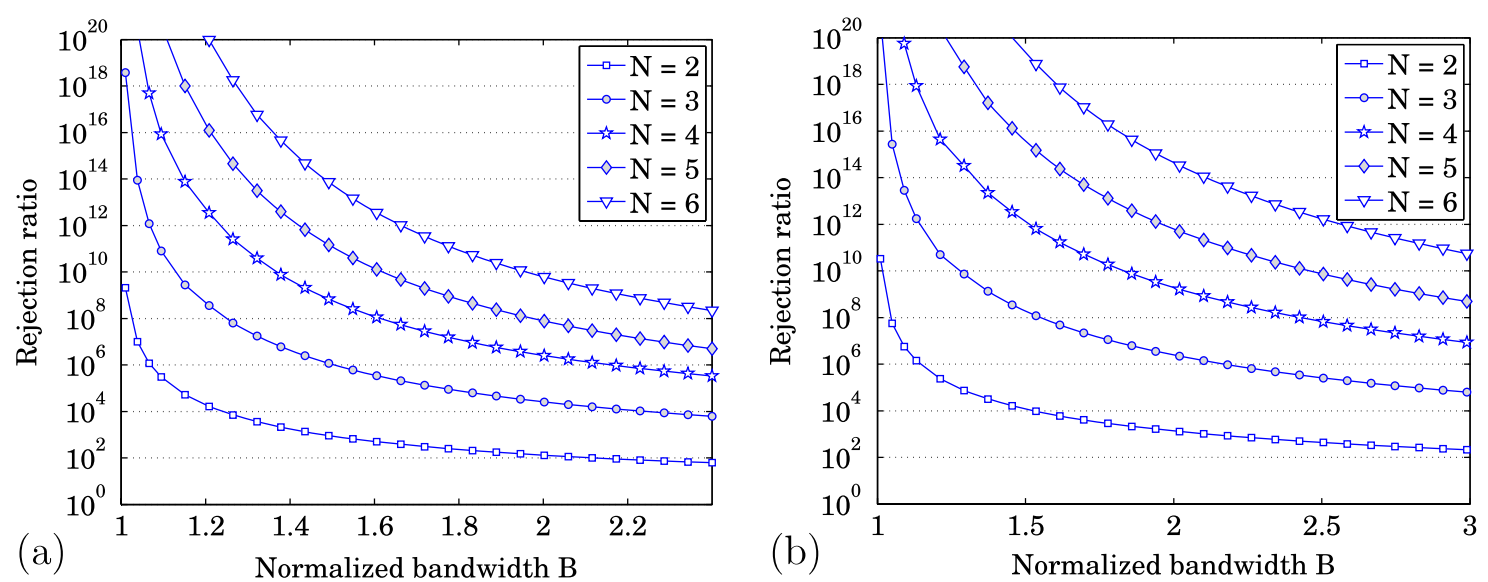

Figure 2. Rejection ratio as a function of the normalized bandwidth $B=\lambda_{\max } / \lambda_{\min }$ for $N \leqslant 6$ (a) in the visible domain and in the near-IR (from $0.4 \mu \mathrm{m}$ to $1 \mu \mathrm{m}, \mathrm{BK} 7$ ) and (b) in the mid-IR (from $6 \mu \mathrm{m}$ to $18 \mu \mathrm{m}, \mathrm{CsI}$ ).

is arbitrarily chosen to be a top-hat function with a normalized bandwidth $B=\lambda_{\max } / \lambda_{\min }$. In the visible and the near-IR regions (from 0.4 to $1 \mu \mathrm{m}$ ), we consider BK7, while in the mid-IR (from 6 to $18 \mu \mathrm{m}$ ), we chose CsI as materials for the plates.

We can see in figures 2 (a) and (b) the rejection ratio as a function of the normalized bandwidth $B$ for $N$ beams $(N \leqslant 6)$. In the visible, we see that a rejection ratio of $10^{5}$ is possible with a spectral bandwidth $B=2.4$ or even wider with four telescopes. A rejection ratio of $10^{6}$ in a broad band with BK7 plates is only possible with a five-beam interferometer. However, although this case is interesting from an experimental point of view, we are mainly interested in the mid-IR region (see figure 2(b)). There we see that a rejection ratio higher than $10^{6}$ is possible over the whole $6-18 \mu \mathrm{m}$ spectral band $(B=3)$ with only four beams. With three beams, a rejection ratio nearly as high as $10^{5}$ can be reached.

In the mid-IR, we chose CsI plates because of the transmission of this material in this spectral band and because of its quasi-linear refractive index. However, other materials can be considered. In figure 3 , we show the rejection ratio for a spectral band from 6 to $18 \mu \mathrm{m}(B=3)$ for $N$ beams $(N \leqslant 6)$ for a few materials that are transparent in the mid-IR. We see that the material leading to the highest rejection ratio is CdTe. This material would allow a rejection ratio of $10^{7}$ over the whole 6-18 $\mu \mathrm{m}$ spectral band with four beams and a rejection ratio of $10^{5}$ with three beams.

This shows that, even though leading to poor performance in the two-beam case, single-glass-plate phase shifters become very interesting when considering three or four beams since they lead to rejection ratios that are high enough for exoplanet detection. Furthermore, this type of phase shifter is very easy to implement and very stable as we will see in the next section.

\subsection{Tolerances}

In the previous section, we have seen that high rejection ratios in a wide spectral band were reachable with a minimum of three beams. In this section, we will show the requirements

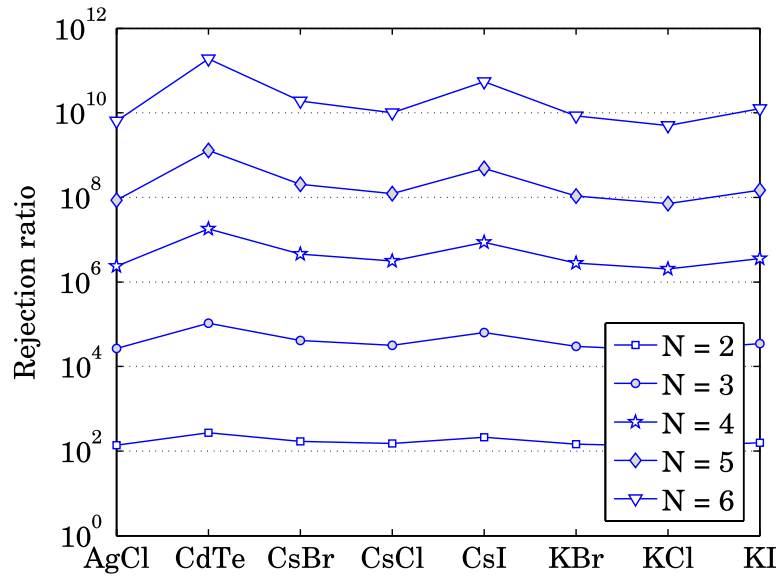

Figure 3. Rejection ratio as a function of the chosen material (indicated in the horizontal axis) in a spectral band ranging from 6 to $18 \mu \mathrm{m}(B=3)$ for $N \leqslant 6$.

in terms of thickness of the glass plates in order to reach these high rejection ratios.

Consider CdTe plates and a spectral band from 6 to $18 \mu \mathrm{m}$. In the three-beam case, our simulations show that the thickness of the additional glass plates should be accurate within $2 \mu \mathrm{m}$ in order to have a $10^{5}$ rejection ratio. If the differential thickness is achieved by rotating $5 \mathrm{~mm}$ thick glass plates, the rotation should be accurate to within $0.3^{\circ}$. This is easily achieved with a standard rotation stage. In the case of a four-beam interferometer, a rejection ratio of $10^{6}$ can be reached if the thickness of the plate is accurate to within $15 \mu \mathrm{m}$, which gives in terms of rotation $2^{\circ}$ of accuracy. This is obviously also very easily achievable.

\section{Experimental results}

In the previous section, we have seen that high rejection ratios were possible with at least three beams. In this section, we will show measurements performed on our table-top two-beam interferometer in order to validate the theoretical predictions 


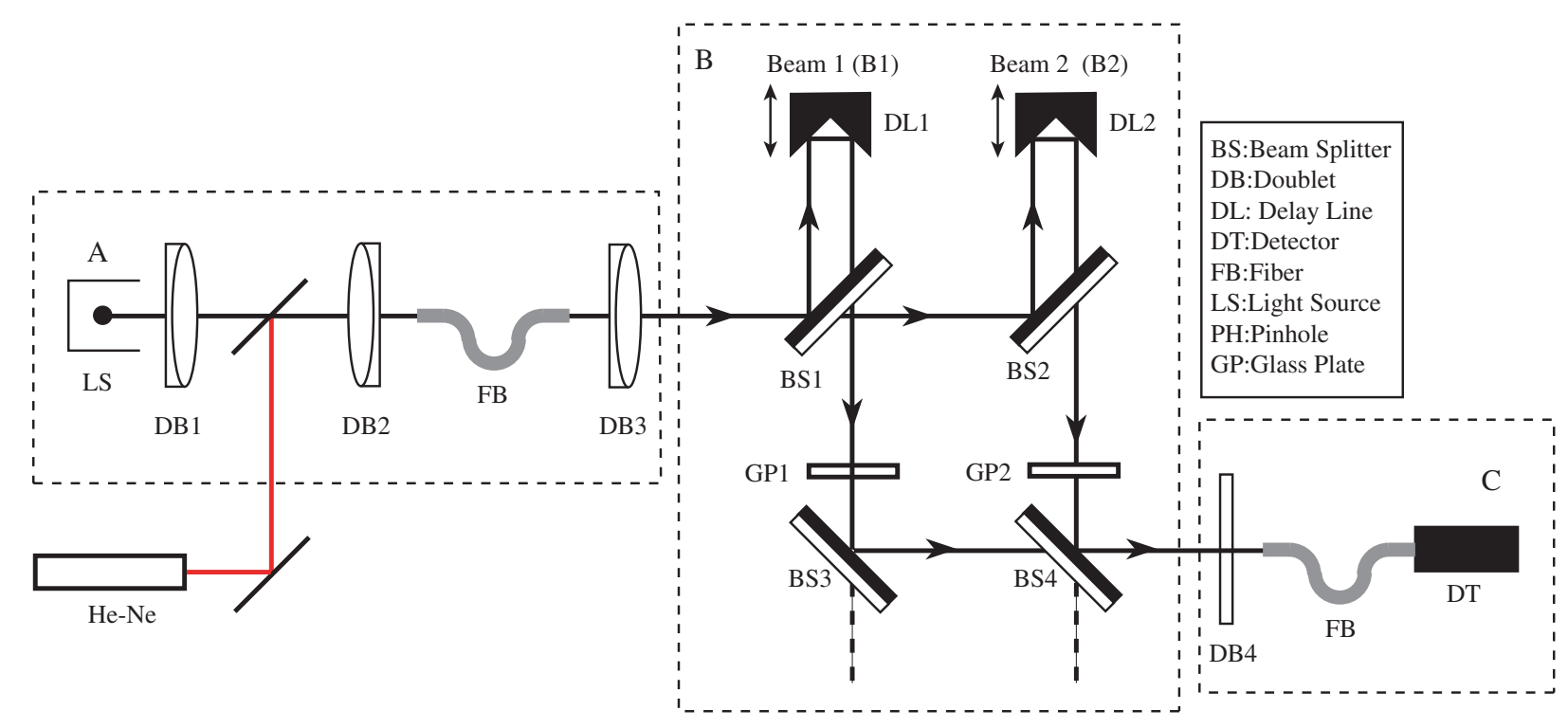

Figure 4. The experimental set-up can be divided into three blocks: the star simulator (A), the interferometer (B) and the detection stage (C).

presented in the previous section. Even though the twobeam case is not the most relevant to Earth-like exoplanet detection since it only allows low rejection ratios, a two-beam measurement is sufficient for demonstrating the theoretical predictions and therefore validating the use of single plates as phase shifters for nulling interferometry. Attempts to validate these predictions have already been conducted in the two-beam case $[14,12]$. However, these results were much lower than the expected values and these measurements only show one point of the rejection ratio as a function of the thickness of the glass plate. Here we will also consider a two-beam experiment and show how dispersion affects the rejection ratio up to the best value possible, given the limitations due to the number of beams and the experiment itself.

\subsection{Set-up}

The experimental set-up is depicted in figure 4. It was originally a modified Mach-Zehnder three-beam interferometer $[15,16]$ where for the demonstration here only two beams were used. The set-up can be divided into three blocks: the star simulator (A), the interferometer (B) and the detection stage (C).

In the star simulator, light from a Xe arc lamp (LS) is focused onto an optical fibre (FB) via achromatic doublets (DB) in order to create a point source. The fibre is also used to decouple the star simulator from the interferometer. This allows these to be mounted on two different optical tables, which reduces drastically the mechanical vibrations and thermal fluctuations, making the interferometer much more stable. Light coming out of the fibre is then collimated. The lamp has an effective spectral bandwidth of $60 \%$ around $600 \mathrm{~nm}$ (at $10 \%$ of the maximal intensity). However, we used an additional filter to cut the long infrared tail, effectively reducing the spectral band to $50 \%$. For alignment purposes, we also use a $\mathrm{He}-\mathrm{Ne}$ laser that we focus onto the same fibre using a folding mirror.
In the interferometer, two beams are created and then recombined with the help of four identical beam splitters. The first beam is reflected by the first beam splitter (BS1) and is sent back through it by a retro-reflector acting as a delay line (DL1). It is then reflected by the third beam splitter (BS3) and finally passes through the fourth beam splitter (BS2). For the second beam, it is transmitted by BS1, reflected by BS2 and sent back through BS2 by another retro-reflector (DL2). The beam is then combined to the first beam after reflection by BS4. The optical path differences between the beams can be varied by changing the position of the delay lines (DL) with piezoactuators. With these delay lines, we can correct optical path differences up to $0.2 \mathrm{~nm}$. However, for this experiment, such an accuracy is not needed since we only try to reach rejection ratios of less than 1000. We therefore used a resolution of $2 \mathrm{~nm}$ for the case of destructive interference and $4 \mathrm{~nm}$ for the lowest rejection ratios.

After recombination, the beams are directed to a singlemode optical fibre for wavefront filtering. The fibre is then connected to a power meter (DT) to detect the outcoming power. The interference pattern is then given by the measured intensity as a function of the position of the delay lines. We define the rejection ratio as the ratio between the maximum and the minimum of the interference pattern.

To achieve high rejection ratios, the amplitudes of the two beams need to be matched achromatically with a so-called amplitude matching device. In our set-up, we used a knifeedge to match the two amplitudes. Even though such a device is not perfectly achromatic since it introduces diffraction, this effect can be neglected when it comes to rejection ratios of less than 1000.

Another parameter to take into account in order to reach the desired rejection ratio is polarization, since a polarization mismatch between the two beams can drastically affect the performance of the nulling interferometer [16]. To match the polarization of the two beams, two Glan laser polarizers have been used: the first one before beam splitting to ensure 


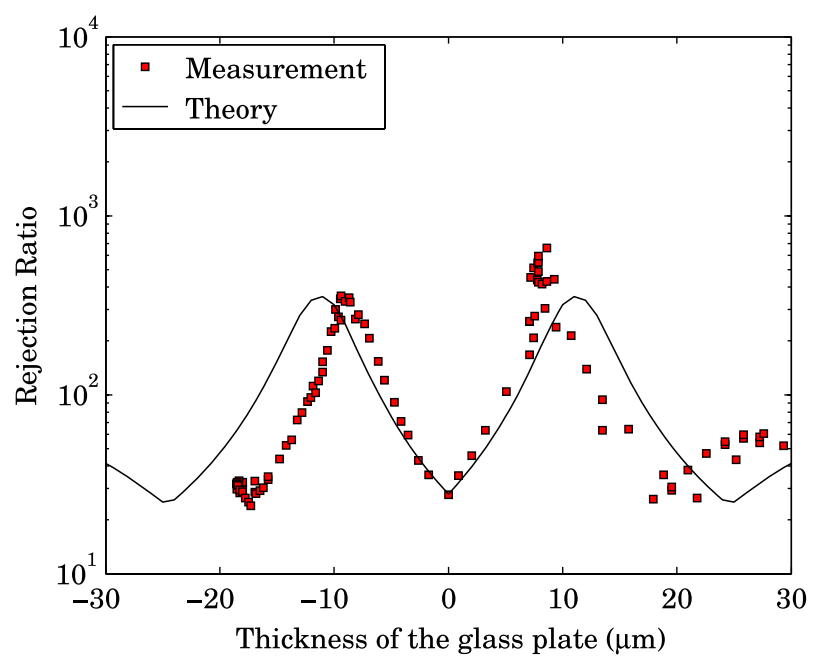

Figure 5. Measured rejection ratio as a function of the thickness of the glass plate $d$.

identical polarization before entering the interferometer and the second one after recombination in order to have an identical polarization state before detection.

In order to see the influence of dispersion on the performance of the interferometer, a glass plate has been introduced in the path of each beam. The required difference in thickness between the two glass plates is of the order of a few microns. For simplicity, we used a $5 \mathrm{~mm}$ thick BK7 plate in each beam that can be rotated in order to achieve the desired differential thickness. The angle of rotation was measured with the help of a laser beam sent onto a mirror fixed on the glass plate. The measurement of the direction of the reflected laser beam is a direct measure of the rotation of the glass plate. From this measurement, we can deduce the effective thickness of the plate using Snell's law and simple geometrical considerations. With our set-up, we could measure differences in this angle of the order of $0.5 \mathrm{mrad}$, which gives typically a thickness accuracy of the order of $0.2 \mu \mathrm{m}$.

\subsection{Measurements}

In figure 5, we show the measurements of the rejection ratio as a function of the differential thickness. Each of the points in this curve corresponds to an interference pattern obtained by scanning the optical path difference between the two beams. The overall shape is very similar to the one expected: a central minimum when there is no differential dispersion and two main peaks when the thickness increases, followed by two secondary minima and secondary maxima. However, we can see that the positions of the maxima and minima do not exactly coincide. The experimental curve seems to be shrunk compared to the theoretical curve. This is probably due to an error in the measurement of the effective thickness of the introduced glass plate. Although we could measure accurately $(0.5 \mathrm{mrad})$ changes in the angles of rotation of the plate, the absolute rotation angle could not be precisely determined (up to a few degrees). This introduces an error in the calculation of the thickness, which can explain the shift in our measurements.
We can also see that for one of the maxima the measured value agrees with the theoretical expectation while, for the other maximum, the rejection ratio is higher than expected. This has been repeatedly measured and remains unexplained. A possible explanation would be the presence of additional non-compensated dispersion induced by the coatings of the beam splitters and the mirrors.

The measured rejection ratio reaches $400-500$ at its maximum. Even though this number is not impressive compared to previous null measurements $\left(8 \times 10^{4}\right.$ in a $32 \%$ bandwidth [17], $10^{6}$ in a $15 \%$ bandwidth and $10^{7}$ in the monochromatic case [18]), the measured rejection ratio is as high as the theory predicts (solid line in figure 5). The only way to improve the rejection ratio in this case is by increasing the number of beams. The goal of the experiment is therefore reached here since the point was to validate the predictions in the two-beam case. The logical next step will be to extend the measurements using a three- or four-beam interferometer.

In figure 6, we can see the recorded interference patterns corresponding to the different minima and maxima of the measured and theoretical rejection ratios. All measured fringes are very similar to the simulated ones. We can see, as expected, that the interference pattern corresponding to the central minimum of the rejection ratio is symmetric with respect to the maximal intensity (see figure 6(a)), while for the two main peaks of the rejection ratio, the fringes are symmetric with respect to the minimal intensity (see figures 6(b) and (c)). The fringes corresponding to the secondary minima start losing their symmetry (see figures 6(d) and (e)). This good agreement between measured and simulated fringes corresponding to the different minima and maxima of the rejection ratio would agree with the fact that the mismatch between measured and theoretical rejection ratio curves would only be due to an error in the measurement of the effective thickness of the glass plate.

\section{Conclusions}

We have studied the effect of dispersion on the performances of $N$-beam nulling interferometers $(N \leqslant 6)$. In the case of two beams, we have seen that we can realize a first-order achromatic phase shift by carefully choosing the thickness of the glass plate. This quasi-achromatic phase shift will lead to an optimal rejection ratio. Increasing the plate thickness further will decrease the rejection ratio to reach a secondary minimum etc. Further, we have seen that in the visible domain (from 0.4 to $1 \mu \mathrm{m}$ ), a rejection ratio of $10^{5}$ is possible with a spectral bandwidth $B=2.4$ or even wider using four telescopes and BK7 plates. A rejection ratio of $10^{6}$ in this spectral band can only be reached with a five-beam interferometer. In the mid-IR, we could reach, using CdTe plates, a rejection ratio of $10^{7}$ over the whole $6-18 \mu \mathrm{m}$ spectral band with four beams and a rejection ratio of $10^{5}$ with three beams. We also investigated the requirements in terms of glass thickness in order to reach these high rejection ratios and we concluded that the requirements can be easily met and the method is therefore robust and stable.

Finally, we have validated our theoretical predictions with measurements performed on our table-top two-beam 


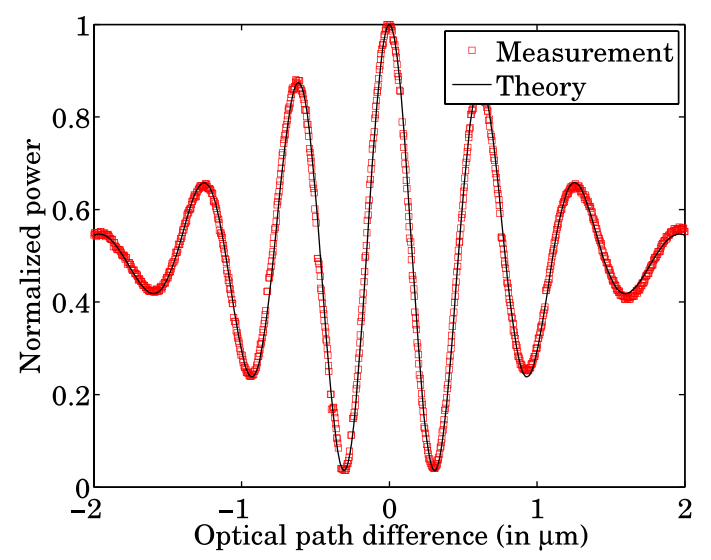

(a)

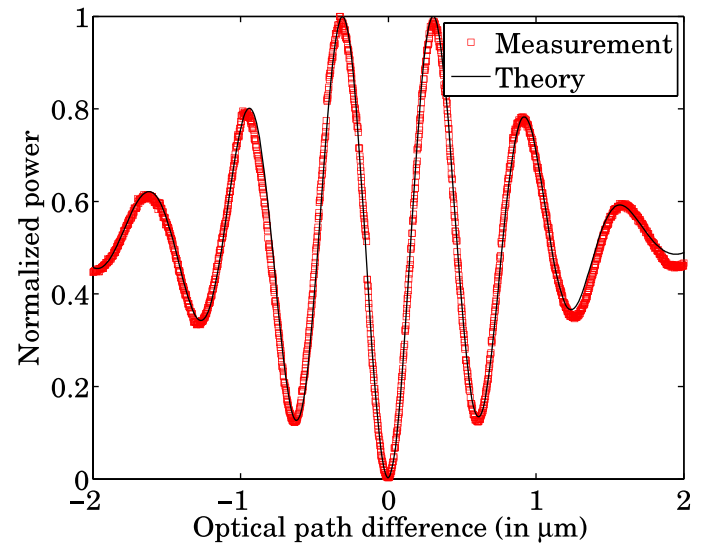

(b)

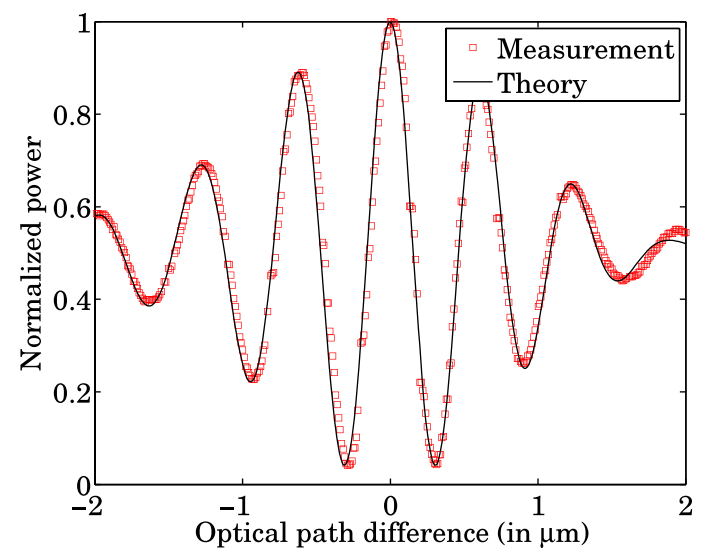

(d)

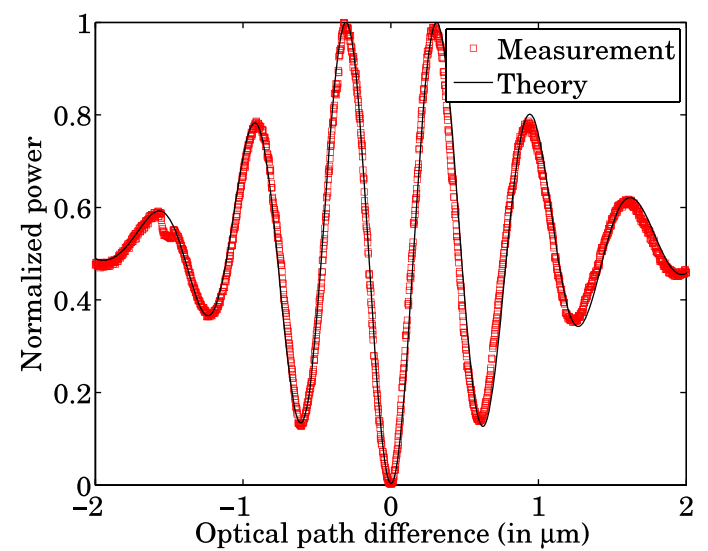

(c)

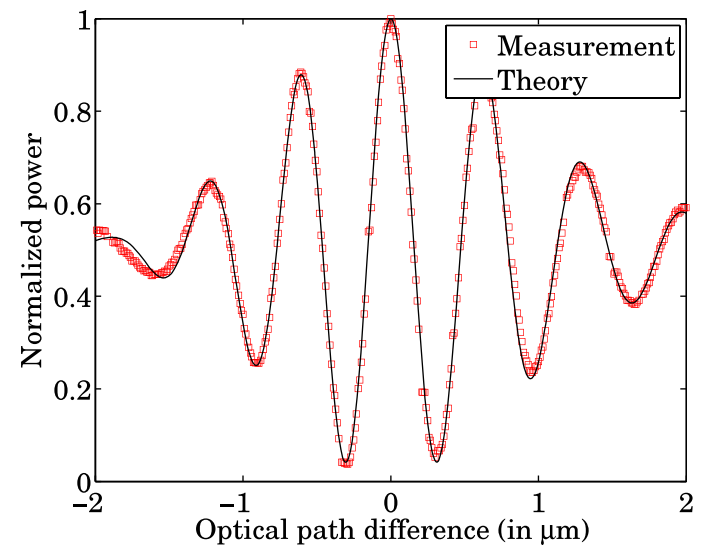

(e)

Figure 6. Measured fringes (individual squares) with a nulling interferometer without an achromatic phase shifter for the following thicknesses of the introduced glass plate: (a) $d=0 \mu \mathrm{m}$, (b) $d=-12 \mu \mathrm{m}$, (c) $d=12 \mu \mathrm{m}$, (d) $d=-24 \mu \mathrm{m}$ and (e) $d=24 \mu \mathrm{m}$. The solid curves show the theoretical results.

interferometer. We measured the rejection ratio as a function of the thickness of the introduced glass plate. The overall shape of the rejection ratio curve was in good agreement with the theory but the positions of the minima and maxima did not exactly coincide. This can be explained by an error in measuring the effective thickness of the glass plate. The measured fringes corresponding to the minima and maxima of the rejection ratio were in very good agreement with the simulated ones, which agrees with the error in the estimation of the thickness. The rejection ratio obtained was theoretically limited to the measured value and could only be improved by increasing the number of beams. We therefore validated the theoretical predictions in the two-beam case.

With this study, we have shown, both theoretically and experimentally, that dispersion plays an important role in exoplanet detection by nulling interferometry. 


\section{Acknowledgment}

This research was supported by TNO Science and Industry.

\section{References}

[1] Bracewell R N 1978 Nature 274 780-1

[2] Fridlund C V M 2000 Darwin-the Infrared Space Interferometer ESA Special Publication vol 451

[3] Beichman C A, Woolf N J and Lindensmith C A 1999 The Terrestrial Planet Finder (TPF): a NASA Origins Program to Search for Habitable Planets

[4] Angel J R, Cheng A Y S and Woolf N J 1986 Nature 232 341-3

[5] Rabbia Y, Gay J, Rivet J-P and Schneider J-L 2003 ESA Special Publication vol 522

[6] Serabyn E and Colavita M M 2001 Appl. Opt. 40 1668-71

[7] Baba N, Murakami N and Ishigaki T 2001 Opt. Lett. 26 1167-9

[8] Mawet D, Baudrand J, Lenaerts C, Moreau V, Riaud P, Rouan D and Surdej J 2004 ESA Special Publication vol 539 pp 519-24
[9] Mieremet A L and Braat J J M 2003 Appl. Opt. 42 1867-75

[10] Spronck J, Pereira S F and Braat J J M 2006 Appl. Opt. 45 597-604

[11] Mieremet A L, Braat J J M, Bokhove H and Ravel K 2000 Proc. SPIE 40061035

[12] Morgan R M, Burge J and Woolf N 2000 Proc. SPIE 4006340

[13] Koresko C D, Mennesson B P, Serabyn E, Colavita M M, Akeson R L and Swain M R 2002 Proc. SPIE 4838 4838-127

[14] Brachet F, Labèque A, Léger A, Ollivier M, Lizambert C, Hervier V, Chazelas B, Pellet B, Lpine T and Valette C 2004 Proc. SPIE 5491991

[15] van der Avoort C, Mieremet C, Pereira S F and Braat J J M 2004 Proc. SPIE $5491808-15$

[16] Spronck J, Pereira S F and Braat J J M 2006 Proc. SPIE $\mathbf{6 2 6 8} 626832$

[17] Peters R, Lay O, Hirai A and Jeganathan M 2007 Proc. SPIE $\mathbf{6 6 9 3} 669315$

[18] Samuele R, Wallace J K, Schmidtlin E, Shao M, Levine B M and Fregoso S 2007 Experimental progress and results of a visible nulling coronagraph IEEE Aerospace Conf. 